\title{
Risk assessment of hepatocellular carcinoma development for indeterminate hepatic nodules in patients with chronic hepatitis B
}

\author{
Haneulsaem Shin ${ }^{1,2}$, Yeon Woo Jung ${ }^{1,2,3}$, Beom Kyung Kim ${ }^{1,2,3}$, Jun Yong Park ${ }^{1,2,3}$, Do Young Kim ${ }^{1,2,3}$, Sang Hoon Ahn ${ }^{1,2,3}$, \\ Kwang-Hyub Han ${ }^{1,2,3}$, Yeun-Yoon $\mathrm{Kim}^{4}$, Jin-Young Choi ${ }^{4}$, and Seung Up Kim ${ }^{1,2,3}$ \\ 'Department of Internal Medicine and ${ }^{2}$ Institute of Gastroenterology, Yonsei University College of Medicine, Seoul; ${ }^{3}$ Yonsei Liver Center, \\ Severance Hospital, Seoul; ${ }^{4}$ Department of Radiology, Yonsei University College of Medicine, Seoul, Korea
}

Background/Aims: A risk prediction model for the development of hepatocellular carcinoma (HCC) from indeterminate nodules detected on computed tomography (CT) $\left(\operatorname{Rad}_{\mathrm{CT}}\right.$ score) in patients with chronic hepatitis B (CHB)-related cirrhosis was proposed. We validated this model for indeterminate nodules on magnetic resonance imaging (MRI).

Methods: Between 2013 and 2016, Liver Imaging Reporting and Data System (LI-RADS) 2/3 nodules on MRI were detected in 99 patients with $\mathrm{CHB}$. The $\operatorname{Rad}_{\mathrm{CT}}$ score was calculated.

Results: The median age of the 72 male and 27 female subjects was 58 years. HCC history and liver cirrhosis were found in $47(47.5 \%)$ and $44(44.4 \%)$ patients, respectively. The median $\operatorname{Rad}_{\mathrm{CT}}$ score was 112 . The patients with $\mathrm{HCC}(\mathrm{n}=41,41.4 \%)$ showed significantly higher $\operatorname{Rad}_{\mathrm{CT}}$ scores than those without (median, 119 vs. 107; $P=0.013$ ); the Chinese university-HCC and risk estimation for HCC in CHB (REACH-B) scores were similar (both $P>0.05$ ). Arterial enhancement, $\mathrm{T} 2$ hyperintensity, and diffusion restriction on MRI were not significantly different in the univariate analysis (all $P>0.05$ ); only the $\operatorname{Rad}_{\mathrm{CT}}$ score significantly predicted HCC (hazard ratio $[\mathrm{HR}]=1.018 ; P=0.007$ ). Multivariate analysis showed HCC history was the only independent HCC predictor ( $\mathrm{HR}=2.374 ; P=0.012)$. When the subjects were stratified into three risk groups based on the $\operatorname{Rad}_{C T}$ score $(<60,60-105$, and $>105)$, the cumulative HCC incidence was not significantly different among them (all $P>0.05$, log-rank test).

Conclusions: $\mathrm{HCC}$ history, but not Rad ${ }_{C T}$ score, predicted CHB-related HCC development from LI-RADS 2/3 nodules. New risk models optimized for MRI-defined indeterminate nodules are required. (Clin Mol Hepatol 2019;25:390-399)

Keywords: Radiographic image interpretation, Computer-assisted; Liver neoplasms; Hepatitis B; Risk assessment; Hepatocellular carcinoma

\begin{abstract}
Abbreviations:
AFP, alpha-fetoprotein; ALT, alanine aminotransferase; AST, aspartate aminotransferase; $\mathrm{AVT}$, antiviral therapy; $\mathrm{CHB}$, chronic hepatitis $\mathrm{B} ; \mathrm{Cl}$, confidence interval; $\mathrm{CT}$, computed tomography; $\mathrm{CU}$, Chinese university; HBeAg, hepatitis B virus envelope antigen; HBV, hepatitis B virus; $\mathrm{HCC}$, hepatocellular carcinoma; HR, hazard ratio; IQR, interquartile range; LI-RADS, Liver Imaging Reporting and Data System; $M R I$, magnetic resonance imaging: $R A D_{c,}$, a risk prediction model for the development of hepatocellular carcinoma from indeterminate nodules detected on computed tomography; REACH-B, risk estimation for hepatocellular carcinoma in chronic hepatitis B
\end{abstract}

Corresponding author: Seung Up Kim

Department of Internal Medicine, Yonsei University College of Medicine, 50-1 Yonsei-ro, Seodaemun-gu, Seoul 03722, Korea

Tel: +82-2-2228-1930, Fax: +82-2-393-6884

E-mail:ksukorea@yuhs.ac

https://orcid.org/0000-0002-9658-8050

\section{Jin-Young Choi}

Department of Radiology, Yonsei University College of Medicine, 50-1 Yonsei-ro, Seodaemun-gu, Seoul 03722, Korea

Tel: +82-2-2228-7400, Fax: +82-2-393-2390

E-mail:gafield2@yuhs.ac

https://orcid.org/0000-0002-9025-6274 


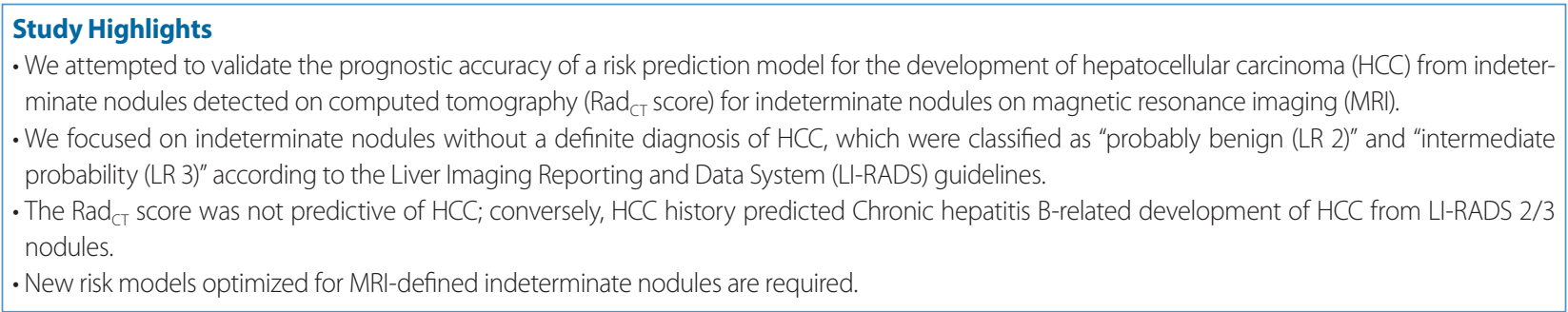

\section{INTRODUCTION}

Liver cancer is the fifth most common cancer in men $(7.9 \%$ of all cancers), theseventh most common cancer in women (6.5\% of all cancers), ${ }^{1}$ and the third most common cause of cancer death worldwide. ${ }^{2}$ In Korea, liver cancer is the second leading cause of malignancy mortality, and $\sim 22.5$ per 100,000 individuals died of hepatocellular carcinoma $(\mathrm{HCC}) .^{3}$ Despite recent progress in follow-ups and treatment strategies, the overall prognosis of patients with HCC has remained dismal (5-year survival rate, $<10 \%$ ) owing to the high proportion of patients with advanced $\mathrm{HCC}^{3}$

If HCC is diagnosed at its late or advanced stage, only palliative treatment, such as trans-arterial chemoembolization, molecular targeted agent administration, or immunotherapy ${ }^{4}$ can be applied, which results in poor survival. However, if HCC is recognized at its early stage, curative treatment options, such as resection, percutaneous ablation, or orthotopic liver transplantation, can be considered, which might yield favorable long-term outcomes. ${ }^{5-8}$ For these reasons, various HCC prediction models, such as the Chinese university (CU)-HCC and risk estimation for HCC in chronic hepatitis $B(C H B)$ (REACH-B) models, have been proposed for early $\mathrm{HCC}$ detection in patients with $\mathrm{CHB}^{2}{ }^{2}$ Recently, magnetic resonance imaging (MRI) using the new liver-specific contrast agent gadoxetic acid (Primovist) have been widely used for HCC diagnosis. ${ }^{9,10}$

Recently, Cho et al. ${ }^{11}$ proposed a risk prediction model for the development of HCC from indeterminate nodules detected on computed tomography (CT) $\left(\operatorname{Rad}_{\mathrm{CT}}\right.$ score) in patients with CHB-related cirrhosis. Unlike the CU-HCC or REACH-B model, this risk model includes radiological findings of "arterial enhancement" on $\mathrm{CT}^{11}$ In their study, indeterminate nodules were categorized into three risk groups with significantly different HCC risks (low-risk group, 1\%; intermediate-risk group, 14.5\%; and high-risk group, $63.1 \%$ at 5 years). The Liver Imaging Reporting and Data System $(\text { LI-RADS })^{12}$ subdivides hepatic nodules detected on CT and MRI in patients at a high risk for HCC based on their radiological characteristics; indeterminate nodules are subdivided as "probably be- nign (LR 2)," "intermediate probability (LR 3)," and "probably HCC $(\mathrm{LR} 4)^{\prime \prime} .^{12}$ In a previous study, patients with CHB-related liver cirrhosis and indeterminate nodules showed significantly higher risks of HCC development. ${ }^{11}$

In this study, we aimed to validate the previously proposed $\operatorname{Rad}_{C T}$ score for indeterminate nodules (LI-RADS 2/3) detected on $\mathrm{MRI}$ and compare its accuracy with those of several conventional risk prediction models for CHB-related HCC development.

\section{PATIENTS AND METHODS}

\section{Patients}

Patients with CHB who had LI-RADS 2/3 lesions on MRI conducted at Yonsei Liver Center, Severance Hospital, Yonsei University College of Medicine from 2013 to 2016 were eligible for inclusion in this retrospective cohort study. CHB was defined as chronic necroinflammatory disease of the liver caused by persistent infection with hepatitis B virus (HBV), with presence of HBV surface antigen in the serum for at least 6 months. LI-RADS 2/3 nodules were defined on the basis of LI-RADS nodules detected on MRI performed for both primary HCC surveillance and diagnosis to characterize liver lesions identified on ultrasonography during HCC surveillance. ${ }^{12-15}$

The exclusion criteria were as follows: (1) HCC development $<6$ months after enrollment; (2) indeterminate nodules related to previously treated lesion; (3) co-infection with hepatitis $C$ and human immunodeficiency virus; (4) active extrahepatic malignancies; (5) other significant medical illness; (6) significant alcohol consumption (>40 g/daily); and (7) insufficient clinical or radiological information for statistical analysis.

The study conformed to the ethical guidelines of the 1975 Declaration of Helsinki and was approved by the institutional review board of each institute. The requirement for written informed consent was waived owing to the retrospective nature of the study. 


\section{Baseline data collection}

Information on age; sex; other co-morbidities, such as hypertension or diabetes mellitus; body mass index; liver cirrhosis ongoing antiviral therapy (AVT); and HCC history, was collected from the medical records. Baseline laboratory parameters, including levels of HBV envelope antigen (HBeAg), HBV DNA, alpha-fetoprotein (AFP), aspartate aminotransferase (AST), alanine aminotransferase (ALT), total bilirubin, and serum albumin and platelet counts, were collected. Radiological findings of nodules, such as maximal size, arterial enhancement, T2 hyperintensity, and diffusion restriction, were also reviewed. The imaging analysis results were interpreted by two expert abdominal radiologists. If the results were different, the final decision was made after agreement between them.

\section{$\operatorname{Rad}_{C T}$ score}

The risk model for the development of HCC from indeterminate nodules was recently proposed by Cho et al. $\left(\operatorname{Rad}_{\mathrm{CT}}\right.$ score). ${ }^{11}$ This score is composed of seven factors: age, enhancement pattern, size, serum albumin level, serum AFP level, HCC history, and $\mathrm{HBeAg}$ level. The formula for the risk score calculation was as follows.

Risk score $=1 \times$ age (years) $+19 \times$ enhancement pattern (arterial non-enhancement or arterioportal shunt $=0$; arterial enhancement $=1)+42 \times \operatorname{size}(\leq 1 \mathrm{~cm}=0 ;>1 \mathrm{~cm}=1)+16 \times$ serum albumin level
(>3.5 g/dL=0; $\leq 3.5 \mathrm{~g} / \mathrm{dL}=1)+31 \times$ serum AFP level $(<100 \mathrm{ng} / \mathrm{mL}=0$; $\geq 100 \mathrm{ng} / \mathrm{mL}=1)+32 \times \mathrm{HCC}$ history (no=0; yes $=1)+18 \times \mathrm{HBeAg}$ level (negative $=0$; positive $=1$ )

Two cutoff values (60 and 105) discriminated the HCC risk into three categories. The 3-year HCC development rate was 1\%, $14.5 \%$, and $53.3 \%$, and the 5-year HCC development rate was $1 \%, 14.5 \%$, and $63.1 \%$ in the low-, intermediate-, and high-risk groups, respectively.

\section{CU-HCC and REACH-B scores}

The CU-HCC score was first derived in a cohort of 1,005 Chinese patients with $\mathrm{CHB}{ }^{16,17}$ It is composed of five factors: age, serum albumin level, total bilirubin level, HBV DNA level, and cirrhosis and ranges from 0 to 44.5. Two cutoff values (5 and 20) discriminated the HCC risk into three categories. The 5-year HCCfree survival rates were $98.3 \%, 90.5 \%$, and $78.9 \%$ in the low-, medium-, and high-risk groups, respectively.

The REACH-B score was derived in 3,584 Chinese patients with CHB from the Taiwanese Risk Evaluation of Viral Load Elevation and Associated Liver (REVEAL) cohort and validated in a cohort of 1,505 patients from three tertiary referral clinics in Hong Kong and South Korea. ${ }^{16,18}$ The variables included in the risk score are sex, age, serum level of ALT, level of HBeAg, and level of HBV DNA. A 17-point risk score was developed, and the HCC risk rate ranged from $0 \%$ to $47.4 \%$ at 5 years and from $0 \%$ to $81.6 \%$ at 10 years for patients with the lowest and highest HCC risks, respectively.

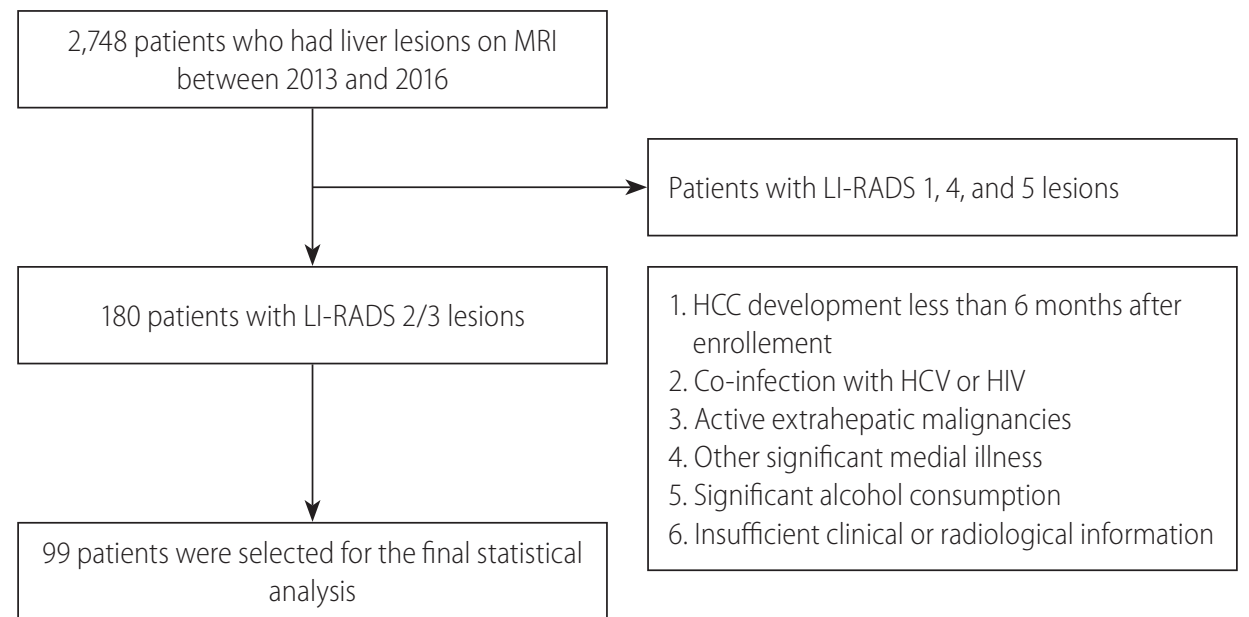

Figure 1. Flow of study population selection. Among 2,748 consecutive patients who underwent liver MRI during the study period, 99 patients were selected for the final statistical analysis. MRI, magnetic resonance imaging; LI-RADS, Liver Imaging Reporting and Data System; HCC, hepatocellular carcinoma; $\mathrm{HCV}$, hepatitis $\mathrm{C}$ virus; $\mathrm{HIV}$, human immunodeficiency virus. 


\section{Follow-up and HCC diagnosis}

Although patients with HCC history were included, all participants were confirmed as being HCC-free at the beginning of the study. Each patient was followed up with AFP examination and ultrasonography, CT, or MRI every 3 or 6 months for HCC surveillance. Most of the patients have been followed up with ultrasonography every 6 months. However, the high-risk patients, for instance, who showed equivocal results based on ultrasonographic findings and AFP level received dynamic imaging according to the physicians' decision. Protocols of CT and MRI are described in Supplementary Material. During the follow-up, data on the actual development to HCC were collected. Two radiologists reviewed the dynamic CT and MRI imaging. HCC diagnosis was based on the radiological findings on CT and/or MRI or on the histological evidence according to the guideline of the American Association for the Study of Liver Diseases. ${ }^{19}$

\section{Statistical analysis}

Student's t-test (or Mann-Whitney test) and the chi-squared test (or Fisher's exact test) were used to compare the baseline characteristics of the patients. In this analysis, we checked the association of HCC occurrence with each variable. The patients were censored at the time of first presentation of HCC or at the last followup. The annual and cumulative incidence of HCC was calculated using the Kaplan-Meier method. To identify the independent risk factors for HCC development, univariate and subsequent multivariate Cox proportional hazard regression analyses were conducted. Hazard ratios (HRs) and corresponding 95\% confidence intervals (Cls) were presented. The final risk score obtained in the $\operatorname{Rad}_{C T}$ and conventional prediction models, including the CU-HCC and REACH-B models, was validated in this cohort. Each risk model was validated via Cox regression analyses. All statistical analysis was performed using the IBM SPSS statistics software, version 24 for Windows (IBM Corp., Armonk, NY, USA).

\section{RESULTS}

\section{Baseline characteristics}

Among 2,748 consecutive patients who underwent liver MRI during the study period, there were 180 patients with LI-RADS 2/3 lesions. After excluding 81 patients, 99 patients were selected for the final statistical analysis (Fig. 1).

The baseline patient characteristics are described in Table 1. The median age was 58 years, and the male sex predominated $(n=72,72.7 \%)$. Liver cirrhosis and HCC history were identified in $44(44.4 \%)$ and $47(47.5 \%)$ patients, respectively. Of the 47 patients who had a previous HCC history, 28 patients received curative treatment (operation [ $n=22]$, radiofrequency ablation $[n=5]$, and cryoablation $[n=1])$ and the other 19 patients received non-

Table 1. Baseline characteristics

\begin{tabular}{|c|c|}
\hline Variable & Value \\
\hline \multicolumn{2}{|l|}{ Demographic variables } \\
\hline Age (years) & $58(51-64)$ \\
\hline Male gender & $72(72.7)$ \\
\hline Hypertension & $76(76.8)$ \\
\hline Diabetes & $77(77.8)$ \\
\hline Body mass index $\left(\mathrm{kg} / \mathrm{m}^{2}\right)$ & $24.5(22.6-26.7)$ \\
\hline Liver cirrhosis & $44(44.4)$ \\
\hline On-going antiviral therapy & $62(62.6)$ \\
\hline Previous HCC history & $47(47.5)$ \\
\hline \multicolumn{2}{|l|}{ Laboratory variables } \\
\hline HBeAg positive & $29(29.3)$ \\
\hline HBV DNA (IU/mL) & $20(20-2,030)$ \\
\hline Alpha-fetoprotein (ng/mL) & $4.8(2.3-8.5)$ \\
\hline Aspartate aminotransferase (IU/L) & $33.0(25.0-44.0)$ \\
\hline Alanine aminotransferase (IU/L) & $27.0(20.0-40.0)$ \\
\hline Total bilirubin (mg/dL) & $0.9(0.7-1.3)$ \\
\hline Serum albumin (g/dL) & $4.2(3.9-4.5)$ \\
\hline Platelet count $\left(10^{9} / \mathrm{L}\right)$ & $127(90-161)$ \\
\hline \multicolumn{2}{|l|}{ Tumor variables } \\
\hline Maximal size $(\mathrm{mm})$ & $11.0(9.0-15.0)$ \\
\hline Arterial enhancement & $16(16.2)$ \\
\hline T2 hyperintensity & $24(24.2)$ \\
\hline Diffusion restriction & $23(23.2)$ \\
\hline \multicolumn{2}{|l|}{ Prediction models } \\
\hline CU-HCC & $6(3-18)$ \\
\hline REACH-B & $8(7-10)$ \\
\hline $\operatorname{RAD}_{\mathrm{CT}}$ score & $112(90-130)$ \\
\hline
\end{tabular}

Variables are expressed as median (interquartile range) or $n(\%)$. HCC, hepatocellular carcinoma; HBeAg, hepatitis B virus envelope antigen; $H B V$, hepatitis $B$ virus; DNA, deoxyribonucleic acid; REACH-B, risk estimation for hepatocellular carcinoma in chronic hepatitis $B$; CU, Chinese university; $R A D_{C T}$, a risk prediction model for the development of hepatocellular carcinoma from indeterminate nodules detected on computed tomography. 
curative treatment (trans-arterial chemoembolization) with the achievement of complete response. The median maximal tumor size was $11.0 \mathrm{~mm}$. Arterial enhancement, T2 hyperintensity, and diffusion restriction were identified in 16 (16.2\%), 24 (24.2\%), and 23 (23.2\%) patients, respectively. The median CU-HCC, $\mathrm{REACH}-\mathrm{B}$, and $\operatorname{Rad}_{\mathrm{CT}}$ scores were 6 (interquartile range $[\mathrm{IQR}]=3$ 18), $8(\mathrm{IQR}=7-10)$, and $112(\mathrm{IQR}=90-130)$, respectively.

\section{Comparison between the patients with and without HCC}

During the follow-up period (median, 20 [IQR=8-29] months), the liver lesions in 41 (41.4\%) patients progressed to HCC. All HCC diagnosis was made according to radiological findings. The comparison of the baseline characteristics between the patients with and without HCC is shown in Table 2. The disease-free survival of patients with HCC was significantly shorter than those

Table 2. Comparison between patients who developed HCC and those who did not

\begin{tabular}{|c|c|c|c|}
\hline Variable & $\begin{array}{l}\text { Patients who developed HCC } \\
\qquad(n=41,41.4 \%)\end{array}$ & $\begin{array}{l}\text { Patients who did not develop } \\
\text { HCC }(n=58,58.6 \%)\end{array}$ & $P$-value \\
\hline \multicolumn{4}{|l|}{ Demographic variables } \\
\hline Age (years) & $59(54-63)$ & $57(50-65)$ & 0.226 \\
\hline Male gender & $30(73.2)$ & $42(72.4)$ & 0.934 \\
\hline Hypertension & $12(29.3)$ & $11(19.0)$ & 0.232 \\
\hline Diabetes & $11(26.9)$ & $11(19.0)$ & 0.354 \\
\hline Body mass index $\left(\mathrm{kg} / \mathrm{m}^{2}\right)$ & $24.1(22.4-26.8)$ & $25.1(22.8-26.4)$ & 0.797 \\
\hline Liver cirrhosis & $13(31.7)$ & $31(53.4)$ & 0.032 \\
\hline On-going antiviral therapy & $28(68.3)$ & $34(58.6)$ & 0.327 \\
\hline Previous HCC history & $27(65.9)$ & $20(34.5)$ & 0.002 \\
\hline \multicolumn{4}{|l|}{ Laboratory variables } \\
\hline HBeAg positive & $11(26.8)$ & $18(31.0)$ & 0.651 \\
\hline HBV DNA (IU/mL) & $20.0(20.0-126.5)$ & $29.5(20.0-52,150.0)$ & 0.399 \\
\hline Alpha-fetoprotein (ng/mL) & $6.8(2.6-14.6)$ & $3.6(2.0-7.1)$ & 0.790 \\
\hline Aspartate aminotransferase (IU/L) & $29(25-40)$ & $36(28-51)$ & 0.031 \\
\hline Alanine aminotransferase (IU/L) & $26(20-39)$ & $29(21-42)$ & 0.044 \\
\hline Total bilirubin (mg/dL) & $0.9(0.6-1.1)$ & $1.0(0.7-1.4)$ & 0.105 \\
\hline Serum albumin (g/dL) & $4.2(3.9-4.6)$ & $4.2(3.9-4.5)$ & 0.526 \\
\hline Platelet count $\left(10^{9} / \mathrm{L}\right)$ & $130(90-163)$ & $120(89-164)$ & 0.709 \\
\hline \multicolumn{4}{|l|}{ Tumor variables } \\
\hline Maximal size (mm) & $10(8-15)$ & $11(9-16)$ & 0.417 \\
\hline Arterial enhancement & $6(14.6)$ & $10(17.2)$ & 0.728 \\
\hline T2 hyperintensity & $13(31.7)$ & $11(19.0)$ & 0.145 \\
\hline Diffusion restriction & $13(31.7)$ & $10(17.2)$ & 0.093 \\
\hline \multicolumn{4}{|l|}{ Prediction models } \\
\hline $\mathrm{CU}-\mathrm{HCC}$ & $3(3-18)$ & $15(3-18)$ & 0.285 \\
\hline REACH-B & $8(7-11)$ & $8(7-10)$ & 0.991 \\
\hline $\mathrm{RAD}_{\mathrm{CT}}$ score & $119(95-145)$ & $107(87-124)$ & 0.013 \\
\hline
\end{tabular}

Variables are expressed as median (interquartile range) or $n(\%)$.

HCC, hepatocellular carcinoma; HBeAg, hepatitis B virus envelope antigen; HBV, hepatitis B virus; DNA, deoxyribonucleic acid; REACH-B, risk estimation for hepatocellular carcinoma in chronic hepatitis $B ; C U$, Chinese university; $R A D_{C \text {, }}$ a risk prediction model for the development of hepatocellular carcinoma from indeterminate nodules detected on computed tomography. 
without (median 30 vs. 46 month, $P<0.001$ ), but the overall survival was not significantly different (median 61 vs. 59 months, $P=0.791)$.

The patients with HCC had significantly higher proportion of liver cirrhosis (68.3\% vs. $58.6 \%$ ) and HCC history (65.9\% vs. $34.5 \%$ ) (both $P<0.05$ ) than those without. They also had significantly lower AST (median, 29 vs. 36 IU/L) and ALT levels (median, 26 vs. $29 \mathrm{IU} / \mathrm{L}$ ) and higher $\operatorname{Rad}_{\mathrm{CT}}$ score (median, 120 vs. 98) than those without (all $P<0.05$ ). The CU-HCC and REACH-B scores were significantly similar between them (all $P>0.05$ ).

\section{Unadjusted HR of the prediction models for HCC development}

In contrast to those of the CU-HCC and REACH-B scores (both $P>0.05)$, the unadjusted $H R$ of the $R A D_{C T}$ score was statistically significant for HCC development $(H R=1.018,95 \% C l=1.005$ 1.031, $P=0.007$ ) (Table 3 ). However, when the subjects were stratified into three risk groups based on the $\operatorname{Rad}_{C T}$ score $(<60$, 60-105, and $>105)$, the cumulative HCC incidence was not significantly different among them (overall $P=0.536$, all $P>0.05$ among adjacent curves, log-rank test) (Fig. 2).

\section{Independent predictors of HCC development}

The Cox regression analysis performed to identify the risk factors associated with development from LI-RADS 2/3 nodules to HCC is shown in Table 4. The univariate analysis revealed that liver cirrhosis, HCC history, and the $R A D_{C T}$ score were associated with HCC development risk (all $P<0.05$ ). In contrast, the MRI parameters, including nodule size, arterial enhancement, $T 2$ hyperintensity, and diffusion restriction, were not predictive of HCC development in the univariate analysis (all $P>0.05$ ). In the subsequent multivariate analysis, HCC history was the only independent predictor of HCC development $(H R=2.374 ; 95 \%$ $\mathrm{Cl}=1.205-4.678 ; P=0.012)$. When the subjects were divided into two groups according to HCC history, the cumulative incidence of HCC was significantly higher in the patients with HCC history than in those without (4.2\% vs. $23.6 \%$ at 1 year; $33.5 \%$ vs. $63.4 \%$ at 3 years; $P<0.001$, log-rank test) (Fig. 3 ).

\section{DISCUSSION}

An accurate assessment of the risk of HCC development is important for establishing individualized strategies of follow-up, in -

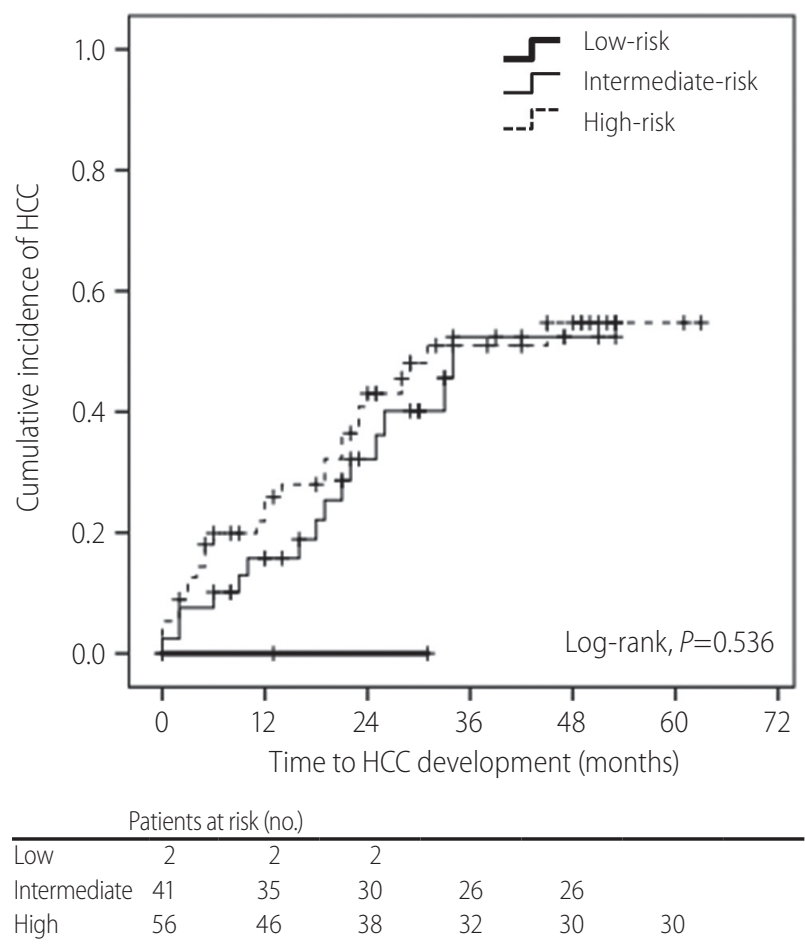

Figure 2. Kaplan-Meier curve for cumulative hepatocellular carcinoma (HCC) incidence according to risk groups based on $\operatorname{Rad}_{\mathrm{CT}}$ score. When the subjects were stratified into three risk groups based on $\operatorname{Rad}_{C T}$ score $(<60,60-105$, and $>105)$, the cumulative HCC incidence was not significantly different among them (all $P=0.536$, log-rank test).

Table 3. Unadjusted hazard ratio of prediction models

\begin{tabular}{lccc}
\hline Prediction model & Hazard ratio & 95\% confidence interval & $P$-value \\
\hline CU-HCC & 0.980 & $0.945-1.046$ & 0.272 \\
REACH-B & 1.026 & $0.923-1.141$ & 0.634 \\
RAD $_{C T}$ Score & 1.018 & $1.005-1.031$ & 0.007
\end{tabular}

CU, Chinese university; HCC, hepatocellular carcinoma; REACH-B, risk estimation for hepatocellular carcinoma in chronic hepatitis $B$; RAD $D_{C T}$ a risk prediction model for the development of hepatocellular carcinoma from indeterminate nodules detected on computed tomography. 
Table 4. Independent predictor of HCC development

\begin{tabular}{|c|c|c|c|c|}
\hline \multirow{2}{*}{ Variable } & \multirow{2}{*}{$\begin{array}{c}\text { Univariate } \\
P \text {-value }\end{array}$} & \multicolumn{3}{|c|}{ Multivariate } \\
\hline & & Hazard ratio & $95 \%$ confidence interval & $P$-value \\
\hline \multicolumn{5}{|l|}{ Demographic variables } \\
\hline Age (years) & 0.201 & - & - & - \\
\hline Male gender & 0.477 & - & - & - \\
\hline Hypertension & 0.069 & - & - & - \\
\hline Diabetes & 0.118 & - & - & - \\
\hline Body mass index $\left(\mathrm{kg} / \mathrm{m}^{2}\right)$ & 0.800 & - & - & - \\
\hline Liver cirrhosis & 0.012 & 0.566 & $0.283-1.132$ & 0.107 \\
\hline On-going antiviral therapy & 0.623 & - & - & - \\
\hline Previous HCC history & 0.002 & 2.374 & $1.205-4.678$ & 0.012 \\
\hline \multicolumn{5}{|l|}{ Laboratory variables } \\
\hline HBeAg positive & 0.620 & - & - & - \\
\hline HBV DNA (IU/mL) & 0.349 & - & - & - \\
\hline Alpha-fetoprotein (ng/mL) & 0.517 & - & - & - \\
\hline Aspartate aminotransferase (IU/L) & 0.057 & - & - & - \\
\hline Alanine aminotransferase (IU/L) & 0.125 & - & - & - \\
\hline Total bilirubin (mg/dL) & 0.150 & - & - & - \\
\hline Serum albumin (g/dL) & 0.522 & - & - & - \\
\hline Platelet count $\left(10^{9} / \mathrm{L}\right)$ & 0.720 & - & - & - \\
\hline \multicolumn{5}{|l|}{ Tumor variables } \\
\hline Maximal size (mm) & 0.207 & - & - & - \\
\hline Arterial enhancement & 0.919 & - & - & - \\
\hline T2 hyperintensity & 0.081 & - & - & - \\
\hline Diffusion restriction & 0.185 & - & - & - \\
\hline
\end{tabular}

HCC, hepatocellular carcinoma; HBeAg, hepatitis B virus envelope antigen; HBV, hepatitis B virus; DNA, deoxyribonucleic acid.

tervention, and management because this ultimately enables the extension of overall survival in patients with $\mathrm{CHB} .{ }^{2}$ To address this issue, several risk prediction models for patients with CHB have been proposed. ${ }^{2,17,20}$ In addition, the importance of dysplastic nodules as precancerous lesions of HCC is well established. Sakamoto et al. found that multistep carcinogenesis is one pathway to HCC development based on 320 resected liver tissues ${ }^{21}$; other investigators also suggested that macroregenerative nodules may represent precancerous lesions. ${ }^{22,23}$ However, because hepatic nodular lesions do not necessarily progress to $\mathrm{HCC}^{24-26}$ it is important to identify true precancerous lesions. Thus, a new risk prediction model $\left(R A D_{C T}\right.$ score) for the development of HCC from indeterminate nodules on $\mathrm{CT}$ in patients with $\mathrm{CHB}$-related cirrhosis has been proposed. ${ }^{11}$

In this study, we attempted to validate the prognostic accuracy of the $R A D_{C T}$ score. Although the unadjusted $H R$ of the $R A D_{C T}$ score was statistically significant $(H R=1.018, P=0.007)$, the cumulative risk of HCC development was not significantly different among the risk groups after adjustment. Instead, HCC history, one of the constituent variables of the $R A D_{C T}$ score, was the only predictor of HCC development. The CU-HCC and REACH-B scores did not significantly predict the risk of HCC development. This finding suggests that the $R A D_{C T}$ score, a new CT-based risk prediction model, and conventional risk models for HBV-related HCC development in patients with CHB-related cirrhosis, might not be applicable for LI-RADS 2/3 nodules on MRI. Thus, further studies are required to optimize the cutoff value for each risk group or establish another MRI-based prediction model.

Our study has several limitations and strengths. First, we validated the predictive performance of the $R A D_{C T}$ score for indeter- 


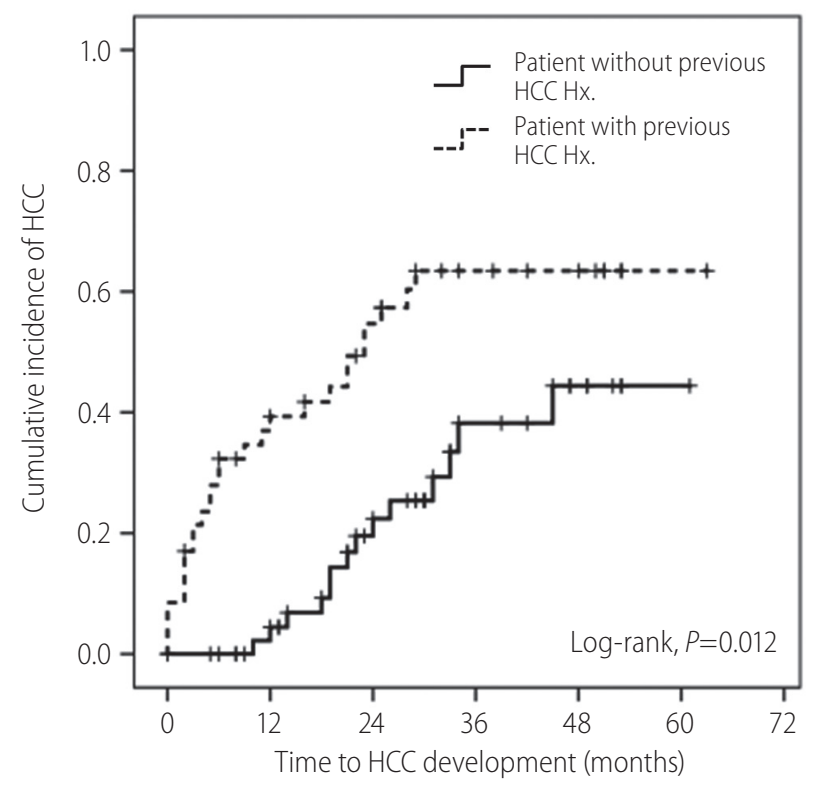

\begin{tabular}{lcccccc}
\multicolumn{7}{c}{ Patients at risk (no.) } \\
\hline HCC Hx. & 52 & 50 & 44 & 35 & 33 & 33 \\
No HCC Hx. & 47 & 36 & 28 & 20 & 20 & 20
\end{tabular}

Figure 3. Kaplan-Meier curve of the cumulative hepatocellular carcinoma (HCC) incidence according to HCC history. HCC history was an independent predictor of HCC development (hazard ratio=2.374; $P=0.012$ ). $H x$. history.

minate nodules in patients with CHB-related cirrhosis and investigated the prognostic accuracy of other conventional risk prediction models. Kobayashi et al. ${ }^{27}$ reported that $18.8 \%$ of 154 nodules in patients with chronic liver disease progressed to $\mathrm{HCC}$ during their follow-up period; the cumulative rate of HCC development at 5 years was $80.0 \%$ for high-grade; $36.6 \%$, low-grade; and $12.4 \%$, regenerative dysplastic nodules. However, because histological assessments for indeterminate liver nodules are generally infeasible, ${ }^{28}$ imaging-based risk assessments, such as use of the $R A D_{C T}$ score, which is composed of routinely available clinical variables, might be appropriate in clinical practice; however, its predictive accuracy was not validated in our study.

Second, we focused on indeterminate nodules without a definite diagnosis of HCC, which were classified as "probably benign (LR 2)" and "intermediate probability (LR 3)" according to the LIRADS guidelines. LR 2/3 nodules can progress to $\mathrm{HCC}{ }^{27}$ However, in contrast to LR 1 nodules without malignancy risks and LR 4/5 nodules with high HCC risks, the probability of HCC development is in the gray zone, with a relatively low risk of HCC development. In our study, 76 out of the 99 patients had only one LI-RADS 2/3 lesion, whereas the others have more than two LI-RADS 2/3 le- sions (two in 16 and three in 7 patients). Although several LIRADS $2 / 3$ lesions can be found simultaneously in a given patient, we considered HCC development as the solid end-points in our study, regardless of the number of LI-RADS 2/3 lesions. Indeed, the aim of our study was to identify the risk factors of HCC development among patients with LI-RADS 2/3 lesions in a clinical point of view, not to identify the risk factors of HCC development among LI-RADS 2/3 lesions in a radiological point of view. Thus, it is important to investigate whether the risk prediction models for LR 2/3 nodules can identify high-risk patients who would benefit from more careful monitoring, which might support the rationale of our study.

Third, although the predictive accuracy was not satisfactory, we tested whether the well-known CU-HCC and REACH-B models have similar prognostic performances when indeterminate nodules exist in patients with CHB. However, these two models were not associated with the risk of HCC development even in the univariate analysis in our cohort; this indicates that the CU-HCC and REACH-B models might not be applicable in this clinical setting. This finding can be explained in several ways. First, despite the low risk of HCC development on MRI, the presence of liver nodules might indicate that the patients already have a higher risk of HCC development than those in the cohorts from which the CUHCC and REACH-B models were derived. Second, because we attempted to validate the $R A D_{C T}$ score, we included patients with HCC history to maintain similar clinical characteristics of the study cohort. Although we excluded patients who developed HCC immediately after enrollment, HCC history is a strong risk factor of HCC development. ${ }^{11,29}$ Indeed, HCC history was the only predictor of HCC development in our study.

There were also several limitations in our study, which remained unresolved. First, the CU-HCC and REACH-B models were basically generated from the cohorts with CHB patients without radiological information regarding the presence of indeterminate hepatic nodules. This might indicate that the CU-HCC and the REACH-B models might have acceptable predictive accuracy, regardless of the potential presence of indeterminate hepatic nodules. Our study focused on only CHB patients with indeterminate hepatic nodules by dynamic imaging, who might be not recognized as having indeterminate hepatic nodules by ultrasound. Thus, our study population might be a subgroup of the whole CHB patients. In addition, due to the different clinical characteristics from the cohorts where CU-HCC and REACH-B models were derived, it might be biased to use CU-HCC and REACH-B models as controls. However, as these two models are well-known pre- 
dictive models, we choose them for comparison.

Second, previous HCC history was selected as a significant predictor of HCC development in our study. Because Khalili et al. ${ }^{30}$ reported that around 20\% among 1-2 cm-sized indeterminate nodules were finally found to be HCC, our results should be interpreted cautiously. Although we included patients who showed HCC-free status for at least 6 months to maximize small sample size, further large-scale studies are warranted to resolve this issue.

Third, despite the large cohort of patients with CHB before exclusion, only a small proportion was selected for the statistical analysis ( $n=99,3.6 \%$ ), and the follow-up period was relatively short (median, 20 [IQR=8-29] months). In addition, owing to the relatively small sample size, the constituent variables of the $R A D_{C T}$ score, such as age, enhancement pattern, size, and serum albumin, serum AFP, and HBeAg levels, were not predictive of development from LI-RADS 2/3 nodules to HCC; this might explain why the $R A D_{C T}$ score was significant only without adjustment.

Fourth, liver cirrhosis was not associated with the risk of $\mathrm{HCC}$ development in our study after adjustment $(P=0.107)$. Although we do not know the exact reason, higher proportion of patients with AVT which can modify fibrotic burden in spite of morphological cirrhosis and small sample size of our cohort might explain this phenomenon.

In conclusion, our study validated the prognostic accuracy of a newly proposed risk prediction model for development of HCC from indeterminate nodules on $C T$ ( $R A D_{C T}$ score); we found that the $\operatorname{Rad}_{\mathrm{CT}}$ score was not predictive of HCC; conversely, HCC history predicted CHB-related development of HCC from LI-RADS 2/3 nodules. New risk models optimized for MRI-defined indeterminate nodules are required.

\section{Authors' contribution}

Conception and design: H Shin, YW Jung, JY Choi, and SU Kim.

Development of methodology: H Shin, YW Jung, JY Choi, and SU Kim.

Analysis and interpretation of data: H Shin, YW Jung, JY Choi, and SU Kim.

Writing, review, and/or revision of the manuscript: All authors.

Administrative, technical, or material support: H Shin, YW Jung, JY Choi, and SU Kim.

Study supervision: SU Kim.

All authors reviewed the paper and approved the final version.

\section{Financial support}

This study was supported by Basic Science Research Program through the National Research Foundation of Korea funded by the Ministry of Science, ICT \& Future Planning (2016R1A1A1A05005138) Ministry of Health and Welfare, Republic of Korea. The funders had no role in study design, data collection and analysis, decision to publish, or preparation of the manuscript.

\section{Conflicts of Interest}

The authors have no conflicts to disclose.

\section{SUPPLEMENTARY MATERIAL}

Supplementary material is available at Clinical and Molecular Hepatology website (http://www.e-cmh.org).

\section{REFERENCES}

1. El-Serag HB. Epidemiology of viral hepatitis and hepatocellular carcinoma. Gastroenterology 2012;142:1264-1273.e1.

2. Lee HW, Ahn SH. Prediction models of hepatocellular carcinoma development in chronic hepatitis B patients. World J Gastroenterol 2016;22:8314-8321.

3. Yoon SK, Chun HG. Status of hepatocellular carcinoma in South Korea. Chin Clin Oncol 2013;2:39.

4. Pardee AD, Butterfield LH. Immunotherapy of hepatocellular carcinoma: unique challenges and clinical opportunities. Oncoimmunology 2012;1:48-55.

5. Raza A, Sood GK. Hepatocellular carcinoma review: current treatment, and evidence-based medicine. World J Gastroenterol 2014:20:4115-4127.

6. Kim BK, Kim SU, Park JY, Kim DY, Ahn SH, Park MS, et al. Applicability of $\mathrm{BCLC}$ stage for prognostic stratification in comparison with other staging systems: single centre experience from long-term clinical outcomes of 1717 treatment-naïve patients with hepatocellular carcinoma. Liver Int 2012;32:1120-1127.

7. Choi SH, Choi GH, Kim SU, Park JY, Joo DJ, Ju MK, et al. Role of surgical resection for multiple hepatocellular carcinomas. World J Gastroenterol 2013;19:366-374.

8. Suh SW, Choi YS. Predictors of micrometastases in patients with Barcelona Clinic Liver Cancer Classification B hepatocellular carcinoma. Yonsei Med J 2017;58:737-742.

9. Hammerstingl R, Huppertz A, Breuer J, Balzer T, Blakeborough A, Carter $R$, et al. Diagnostic efficacy of gadoxetic acid (Primovist)- 
enhanced MRI and spiral CT for a therapeutic strategy: comparison with intraoperative and histopathologic findings in focal liver lesions. Eur Radiol 2008;18:457-467.

10. Kim SH, Kim SH, Lee J, Kim MJ, Jeon YH, Park Y, et al. Gadoxetic acid-enhanced MRI versus triple-phase MDCT for the preoperative detection of hepatocellular carcinoma. AJR Am J Roentgenol 2009;192:1675-1681.

11. Cho HJ, Kim B, Lee JD, Kang DR, Kim JK, Lee JH, et al. Development of risk prediction model for hepatocellular carcinoma progression of indeterminate nodules in hepatitis B virus-related cirrhotic liver. Am J Gastroenterol 2017;112:460-470.

12. An C, Rakhmonova G, Choi JY, Kim MJ. Liver imaging reporting and data system (LI-RADS) version 2014: understanding and application of the diagnostic algorithm. Clin Mol Hepatol 2016;22:296-307.

13. Korean Liver Cancer Study Group and National Cancer Center, Korea. Practice guidelines for management of hepatocellular carcinoma 2009. Korean J Hepatol 2009;15:391-423.

14. European Association For The Study Of The Liver; European Organisation For Research And Treatment Of Cancer. EASL-EORTC clinical practice guidelines: management of hepatocellular carcinoma. J Hepatol 2012;56:908-943.

15. Mitchell DG, Bruix J, Sherman M, Sirlin CB. LI-RADS (Liver Imaging Reporting and Data System): summary, discussion, and consensus of the LI-RADS Management Working Group and future directions. Hepatology 2015;61:1056-1065.

16. Wong GL, Wong VW. Risk prediction of hepatitis B virus-related hepatocellular carcinoma in the era of antiviral therapy. World J Gastroenterol 2013;19:6515-6522.

17. Wong VW, Chan SL, Mo F, Chan TC, Loong HH, Wong GL, et al. Clinical scoring system to predict hepatocellular carcinoma in chronic hepatitis B carriers. J Clin Oncol 2010;28:1660-1665.

18. Yang HI, Yuen MF, Chan HL, Han KH, Chen PJ, Kim DY, et al. Risk estimation for hepatocellular carcinoma in chronic hepatitis $B$ (REACHB): development and validation of a predictive score. Lancet Oncol 2011;12:568-574.

19. Heimbach JK, Kulik LM, Finn RS, Sirlin CB, Abecassis MM, Roberts $L R$, et al. AASLD guidelines for the treatment of hepatocellular carcinoma. Hepatology 2018;67:358-380.

20. Shim JJ, Oh IH, Kim SB, Kim JW, Lee CK, Jang JY, et al. Predictive value of antiviral effects in the development of hepatocellular carcinoma in the general Korean population with chronic hepatitis B. Gut Liver 2016;10:962-968.

21. Sakamoto M, Hirohashi S, Shimosato Y. Early stages of multistep hepatocarcinogenesis: adenomatous hyperplasia and early hepatocellular carcinoma. Hum Pathol 1991;22:172-178.

22. Theise ND, Schwartz M, Miller C, Thung SN. Macroregenerative nodules and hepatocellular carcinoma in forty-four sequential adult liver explants with cirrhosis. Hepatology 1992;16:949-955.

23. Hytiroglou P, Theise ND, Schwartz M, Mor E, Miller C, Thung SN. Macroregenerative nodules in a series of adult cirrhotic liver explants: issues of classification and nomenclature. Hepatology 1995;21:703-708.

24. Terasaki S, Kaneko S, Kobayashi K, Nonomura A, Nakanuma Y. Histological features predicting malignant transformation of nonmalignant hepatocellular nodules: a prospective study. Gastroenterology 1998;115:1216-1222.

25. Seki S, Sakaguchi H, Kitada T, Tamori A, Takeda T, Kawada N, et al. Outcomes of dysplastic nodules in human cirrhotic liver: a clinicopathological study. Clin Cancer Res 2000;6:3469-3473.

26. Borzio M, Fargion S, Borzio F, Fracanzani AL, Croce AM, Stroffolini T, et al. Impact of large regenerative, low grade and high grade dysplastic nodules in hepatocellular carcinoma development. J Hepatol 2003;39:208-214.

27. Kobayashi M, Ikeda K, Hosaka T, Sezaki H, Someya T, Akuta N, et al. Dysplastic nodules frequently develop into hepatocellular carcinoma in patients with chronic viral hepatitis and cirrhosis. Cancer 2006;106:636-647.

28. Seeff LB, Everson GT, Morgan TR, Curto TM, Lee WM, Ghany MG, et al. Complication rate of percutaneous liver biopsies among persons with advanced chronic liver disease in the HALT-C trial. Clin Gastroenterol Hepatol 2010;8:877-883.

29. Colecchia A, Schiumerini R, Cucchetti A, Cescon M, Taddia M, Marasco $G$, et al. Prognostic factors for hepatocellular carcinoma recurrence. World J Gastroenterol 2014;20:5935-5950.

30. Khalili K, Kim TK, Jang HJ, Yazdi LK, Guindi M, Sherman M. Indeterminate 1-2-cm nodules found on hepatocellular carcinoma surveillance: biopsy for all, some, or none? Hepatology 2011;54:20482054. 